\title{
Validation of a Novel Geometric Coordination Registration using Manual and Semi-automatic Registration in Cone-beam Computed Tomogram
}

\author{
Walter Y.H. Lam ${ }^{1}$, Henry Y.T. Ngan ${ }^{2}$, Richard T.C. Hsung ${ }^{1}$, \\ Henry W.K. Luk', Tazuko K. Goto ${ }^{3}$, Edmond H.N. Pow ${ }^{1}$ \\ ${ }^{1}$ Faculty of Dentistry, The University of Hong Kong, Hong Kong SAR, China \\ ${ }^{2}$ Department of Mathematics, Hong Kong Baptist University, Hong Kong SAR, China \\ ${ }^{3}$ Department of Oral and Maxillofacial Radiology, Tokyo Dental College, Japan
}

\begin{abstract}
Cartesian coordinates define on a physical cubic corner (CC) with the corner tip as the origin and three corresponding line angles as ( $x, y, z$ )-axes. In its image (virtual) domains such as these obtained by cone-beam computed tomography (CBCT) and optical surface scanning, a single coordinate can then be registered based on the $C C$. The advantage of using a $C C$ in registration is simple and accurate physical coordinate measurement. The accuracy of image-to-physical (IP) and imageto-image (II) transformations, measured by target registration error (TRE), can then be validated by comparing coordinates of target points in the virtual domains to that of the physical control. For the $C B C T$, the registration may be performed manually using a surgical planning software SimPlant Pro (manual registration (MR)) or semi-automatically using MeshLab and 3D Slicer (semiautomatic registration (SR)) matching the virtual display axes to the corresponding $(x-y-z)$-axes. This study aims to validate the use of CC as a surgical stereotactic marker by measuring TRE in MR and $S R$ respectively. Mean TRE is $0.56+/-0.24 \mathrm{~mm}$ for $M R$ and $0.39+/-0.21 \mathrm{~mm}$ for $S R$. The $S R$ results in a more accurate registration than the $M R$ and point-based registration with 20 fiducial points. TRE of the MR is less than $1.0 \mathrm{~mm}$ and still acceptable clinically.
\end{abstract}

\section{Introduction}

In oral implant surgery, dental surgeons aim to place titanium implants in patient's jaw bone at a position that facilitate biological and esthetic tooth replacement [1]. CBCT is a type of computed tomography (CT) with cone shape $\mathrm{x}$-ray beam and has been used to assess the bone bed for the implant placement before the surgery. It has the advantage of faster image acquisition and reduced radiation to the patient that conventional CT $[2,3]$. A three-dimensional (3D) grey-scale image and orthogonal crosssectional (sagittal, coronal and horizontal) views of patient's jaw bone in the selected field of view (FOV) will be generated and from this, then surgeons can plan and place virtual implants in surgical planning software such as SimPlant Pro. Real time computer-assisted dynamic or template-based static surgical guidance have been developed to facilitate accurate implant placement by transferring this pre-operative virtual planning.

The current method of the clinical assessment of the accuracy of guided implant surgery is by taking pre- and post- implant placements in CBCT. Then, the relative positional difference between the planned and placed implant positions is determined by a best fit algorithm superimposition [4]. This is an intensity-based registration and sub-voxel (voxel is imaging unit of $\mathrm{CBCT}$ ) accuracy can be achieved automatically with respect to the stereotactic reference [5]. However, any imaging modality has its inherent acquisition error and does not truly represent the actual physical object [6]. The linear and 3D measuring accuracies of CBCT have been found to have mean errors of $0.16 \mathrm{~mm}$ and 1.07 $\mathrm{mm}$ respectively in comparison to physical object [7]. This may complicate the calibration of the image registration. There is a need of a stereotactic reference that allows simple linear and coordinate measurement of physical and virtual domains, thereby determine the relative error of image acquisition and registration.

Stereotactic surgery has been transitioned from frame-based, such as Horsley-Clarke apparatus invented by Horsley et al. in 1908 and N-localizer proposed by Brown et al. in 1979, to frameless [8]. The coordinate system in the stereotactic frames was defined by attaching the frame to human body in a fixed position and registering to virtual imaging. In frameless systems, the coordinate was defined by anatomical landmarks or artificial markers and these points were then identified manually or automatically in image-guided surgery. This point-based registration is not precise enough and divergences of $2-5 \mathrm{~mm}$ has been reported clinically. Currently, clinical negligible difference in sample size is commonly regarded as $0.2 \mathrm{~mm}$. This paper therefore only suggests a $2 \mathrm{~mm}$ safety margin for errors. Later, a surfacebased registration of the patient position has been proposed and patient surface is traced with optical equipment and matched with the preoperative data. Moreover, temporary insertion of screws into patient's bone for registration may be justified for major surgeries but too invasive for oral implant surgery.

TRE is preferred to fiducial registration error (FRE) in the determination of the registration accuracy [9]. TRE is the distance between homologous points other than the centroids of fiducials while FRE is the root mean square distance between homologous fiducials after registration. Physically, locating and identifying the centroid of a fiducial point may be difficult i.e. fiducial location error (FLE), and in point-based registration, this will contribute to registration error since it has been shown that $\mathrm{TRE}^{2}$ is roughly proportional to $\mathrm{FLE}^{2}$. Physical determination of the centroid (origin) would be difficult with $0.3 \mathrm{~mm}$ error in physical domain and $0.4 \mathrm{~mm}$ in computed tomography (CT) and estimated TRE of 0.5-0.6 mm when 4 ball markers was used [10].

In this study, a $\mathrm{CC}$ as stereotactic fiducial reference is proposed to be used with its point angle representing the origin $O$ and three line angles representing the (x, y, z)-axes. Three orthogonal surfaces forming this corner representing $(\mathrm{x}, \mathrm{y}, \mathrm{z})$ planes. For the image registration, the $(\mathrm{x}, \mathrm{y}, \mathrm{z})$-surfaces of the real physical $\mathrm{CC}$ is mapped to the orthogonal (sagittal, coronal and horizontal) views in the virtual image domain. Ideally, this registration should be automatically/semi-automatically performed to reduce errors in MR [11]. It is unknown whether human errors in image registration are clinically significant in which $2 \mathrm{~mm}$ safety margin has been suggested for oral implant surgery [12]. SimPlant Pro (version 16.0) is an implant planning software for 
clinicians to virtually plan for the placement of titanium implant. By manually mapping orthogonal view surfaces of the SimPlant Pro to the (x,y, z)-planes of the CC, MR is completed [13]. Alternatively, SR is possible in software such as MeshLab (version 1.3.2) and 3D Slicer (version 4.3.1), in which some of manual procedures may be performed automatically. TRE has been used for the calibration of the use of $\mathrm{CC}$ in image registration.

This paper is organized as follows. Materials and Method is presented in Section 2. Results of experiments are given in Section 3. Discussion is offered in Section 4. Lastly, Conclusion is drawn in Section 5.

\section{Materials and Methods}

The objective of this study is to validate the use of $\mathrm{CC}$ as a surgical stereotactic marker, so as to determine the TRE when the physical CC is used as a stereotactic reference in image registration of CBCT. Registration process can be performed either manually or semi-automatically. Each point is assigned with Cartesian coordinates with reference to the $\mathrm{CC}$ in the physical and corresponding image domains. TRE is the difference of coordinates of homologous points in these domains. Cartesian coordinates are essential tangential linear distance of a point to ( $\mathrm{x}$, $\mathrm{y}, \mathrm{z}$ )-axes. Linear measurement error between two points in the $\mathrm{CBCT}$ is also determined and its contribution in TRE can be estimated.

Six plastic (acrylic) cubes $(80 \mathrm{~mm} \times 80 \mathrm{~mm} \times 40 \mathrm{~mm})$ were machine milled (Figure 1). One corner tip was defined as the origin $O$ of the Cartesian coordinates and the three line angles forming the corner of the origin was defined as $(\mathrm{x}, \mathrm{y}, \mathrm{z})$-axes. Radiopaque Barium Sulphate $\left(\mathrm{BaSo}_{4}\right)$ varnish was painted on the $(\mathrm{x}, \mathrm{y}, \mathrm{z})$ planes of corner (each $10 \mathrm{~mm} \times 10 \mathrm{~mm}$ ) for its identification in CBCT. Five radiopaque point markers $(1 \mathrm{~mm}$ diameter $)$ were distributed and stuck on each plane.

CBCTs (iCAT classic 3D imaging system, Imaging Science International Inc., Hatfield, PA, USA) were taken with a protocol of the largest FOV and highest available resolution selected, i.e. full $13 \mathrm{~cm} 40$ seconds 0.25 voxel high resolution. A total of 599 frames of $2 \mathrm{D}$ planar images were acquired. The reconstructed data

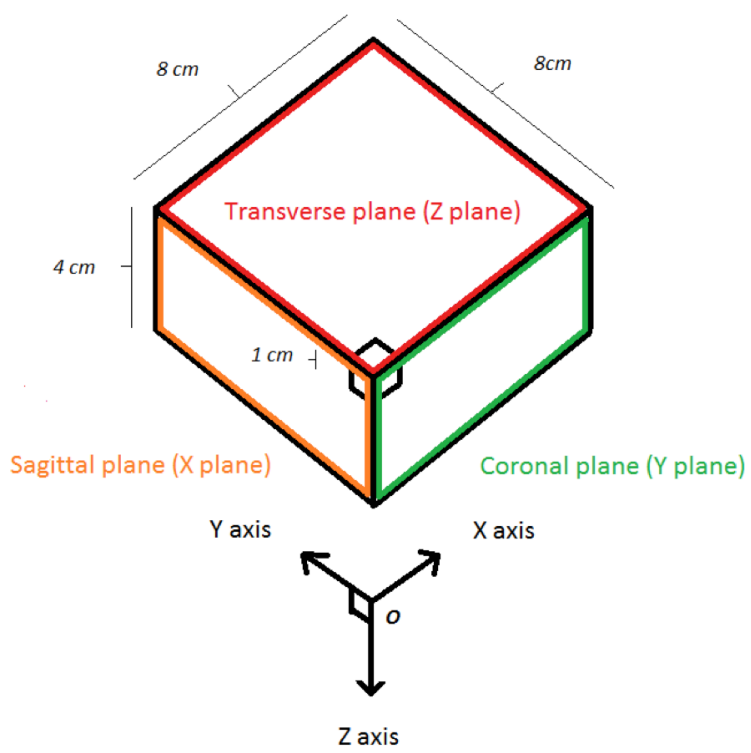

Figure 1. Physical acrylic cube, its corner surfaces and line angles representing the origin, $x-y-z$ planes and axes. in Digital Imaging and Communications in Medicine (DICOM) format were loaded into two personal computers. The first specification is Dell Precision T1700 workstation with a graphic card NVIDIA Quadro K2000 and a 23-inch Dell P2314H LED monitor with a screen resolution of $1920 \times 1080$ installed with SimPlant Pro (version 16.0, Materialise NV, Leuven, Belgium) for the MR. Another personal computer is equipped with IBM ThinkStation D20, Intel(R) Xeon(R) CPU E5645@2.40GHz, 2395 Mhz, 6 Cores, 12 Logical Processors, 16.0 GB Installed Physical Memory (RAM) with a graphic card NVIDIA Quadro 4000) installed with MeshLab (version 1.3.3 [16]) and 3D Slicer (version 4.3.1 [17]) for the SR.

Since the proposed $\mathrm{CC}$ is a rigid body and the registration is based on a point-based system on fiducial points, therefore the accuracy of the registration means how much errors can be found. Basically, a point-based registration can be understood as a $3 \mathrm{D}$ translation $T$ and rotation $R$ to match one set of $n$ points of $A=\left(a_{i}\right)_{i=1, \ldots, n}$ and another set of respective points $B=$ $\left(b_{i}\right)_{i=1, \ldots, n}$.

Linear distances between two points were measured (i.e. measurement error) and the tangential linear distances of each point marker to the (x, y, z)-axes were measured. The root-meansquare (RMS) distances, (denoted as $R M S_{\text {dist }}$ ) of the Cartesian coordinate (i.e. tangential distances to the (x,y, z)-axes) of a point in its physical and virtual domain were calculated.

$$
\begin{array}{r}
R M S_{d i s t}=\sqrt{\left(\frac{\sum_{i=1}^{n} d_{i}^{2}}{n}\right)} \\
\text { and } d_{i}=\sqrt{\left(D_{x i}^{2}+D_{y i}^{2}\right)}
\end{array}
$$

where $d_{i}$ is difference in distance of a specific point in physical and virtual domain at time $i$. $D_{x i}$ is the difference in x-coordinate and $D_{y i}$ is the difference in y-coordinate (in $\mathrm{Z}$ plane). This $R M S_{\text {dist }}$ is usually to incorporate with the minimization of the FRE. Herein, the registration goal is to minimize the FRE [8] as

$$
F R E^{2}=\frac{1}{n} \sum_{i=1}^{n}\left|R a_{i}+T-b_{i}\right|^{2}
$$

In an ideal case, the perfect fiducial registration is $F R E=0$.

The traditional theoretical accuracy in image registration is defined by FLE [8] that means the distance between the unknown real fiducial position and the localized point before any alignment. If there are $k$ components of errors which are independent and orthogonal, then

$$
\left\langle F L E^{2}\right\rangle=k \epsilon^{2} \sigma^{2}
$$

where $\epsilon \mathrm{s}$ a smallness parameter in the perturbation theory and $\sigma$ is from a normal distribution $N(0, \sigma)$. When $k=3$ in our case,

$$
\left\langle F R E^{2}\right\rangle=\left(1-\frac{2}{n}\right)\left\langle F L E^{2}\right\rangle
$$

In perfect registration, the coordinates of a point should be identical in both physical and virtual domains and therefore any distance between them will be the target registration error (TRE) of the image registration.

In general, the TRE at a spatial 3D position of the (x,y, z)axes can be denoted as $\operatorname{TRE}(p)$ that indicates the distance between the virtual computerized domain and real physical domain on the CC. In $[8]$,

$$
T R E^{2}(p)=|R p+T-p|^{2}
$$

Physical measurements were performed using a caliper and this is the gold standard of this study. Physical and virtual (MR (Figure 2) and SR (Figure 3)) measurements were performed by three independent assessors respectively. Differences between the physical and virtual measurements and the TREs were calculated and analyzed with one sample t-test at significance level 0.05. 


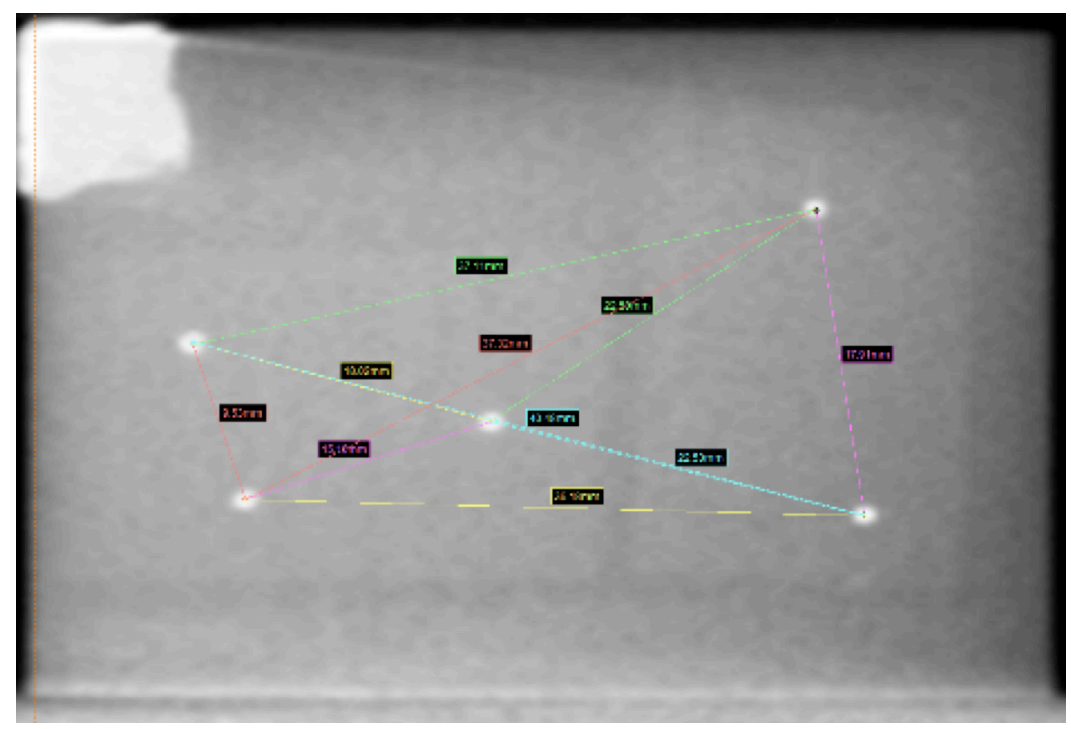

(a)

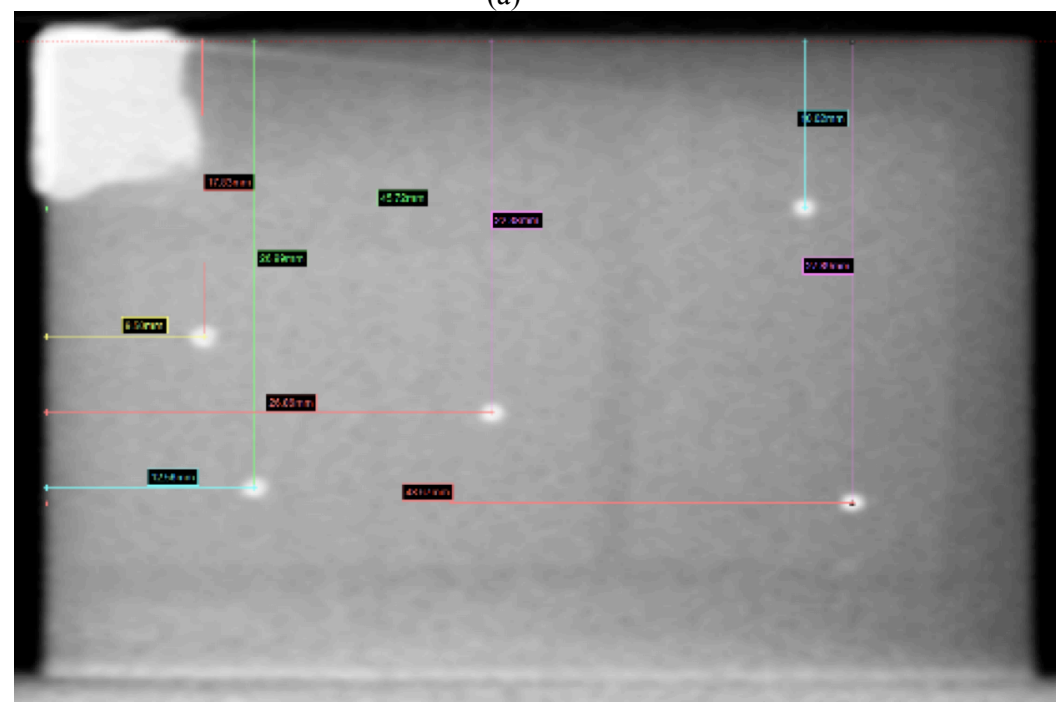

(b)

Figure 2. (a) Linear distance measurement in SimPlant Pro; (b) Coordinate measurement (tangential linear distance to axes). The radiopaque upper left corner was defined as the origin of the Cartesian coordinate. This corresponding to Y plane in Figure 1.

Sample size calculation. Based on the preliminary study [13], standard deviation for distance and coordinate measurement were $0.27 \mathrm{~mm}$ and $0.23 \mathrm{~mm}$ respectively, therefore 22 samples are needed for equivalence test $\alpha=0.05$ and $90 \%$ power.

\section{Results}

\section{Measurement error}

Mean linear distance differences (i.e. mean linear distance measurement error) between two point markers was found to be $0.19 \mathrm{~mm}$ (SD 0.15) for SimPlant Pro (MR) and $-0.10 \mathrm{~mm}$ (SD 0.15 ) for MeshLab and 3D Slicer (SR) in comparison to physical gold standard (Table 1). Mean virtual linear measurements were greater than corresponding mean physical linear measurements. One sample t-test found significant difference to zero for linear measurement error in both MR and SR $(P=0.000)$.

Even in perfect registration, linear measurement error may result in coordinate discrepancy and therefore "registration error" (TRE) of $0.21 \mathrm{~mm}$ for the MR and $0.14 \mathrm{~mm}$ for the SR (i.e. square root of the sum of square of linear measurement in $(x, y, z)-$ planes)).

For example, in a perfect registration, the measurement error for a point's coordinate in an $\mathrm{x}$-plane, the $\mathrm{y}$ and $\mathrm{z}$ coordinates may reveal error of square root $\left(0.19^{2}+0.19^{2}\right)$ for MR and square root of $\left(0.10^{2}+0.10^{2}\right)$ for SR.

\section{Registration error}

Tangential distances of point markers to $(x, y, z)$-axes (i.e. coordinates) were measured and the discrepancy in coordinates of physical to virtual measurements are presented in Table 2. Mean tangential distances to ( $\mathrm{x}, \mathrm{y}, \mathrm{z}$ )-axes in MR and to (x, y)-axes in SR were significantly smaller than physical measurement (one sample t-test; $\mathrm{P}=0.00$ to 0.000 ). For MR, significant difference was found between blocks ( $\mathrm{Z}$ coordinate second measurement $\mathrm{B}$ and $\mathrm{F}$ ) $(P<0.008)$. For SR, significant difference was found between blocks (x-coordinate first measurement $\mathrm{A}$ and $\mathrm{B}, \mathrm{A}$ and $\mathrm{C}, \mathrm{A}$ and $\mathrm{D}, \mathrm{B}$ and $\mathrm{E}, \mathrm{B}$ and $\mathrm{F}, \mathrm{C}$ and $\mathrm{E}, \mathrm{C}$ and $\mathrm{F}, \mathrm{D}$ and $\mathrm{E}, \mathrm{D}$ and $\mathrm{F} ; \mathrm{Y}$ 

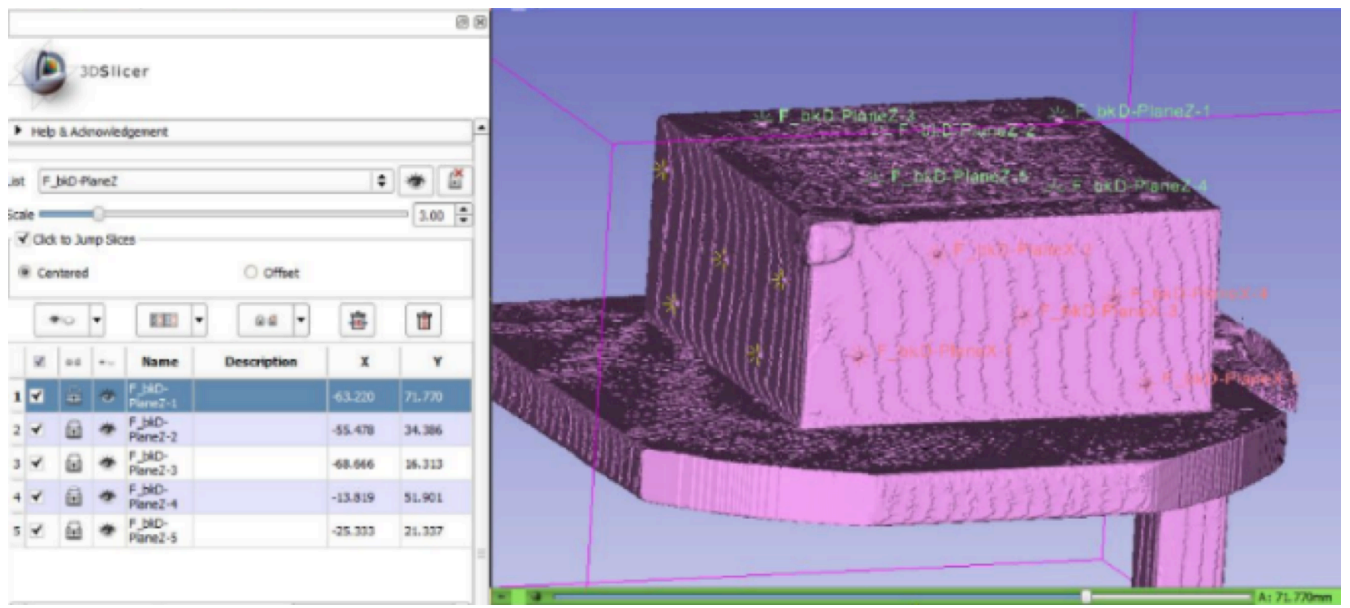

Figure 3. Coordinate measurement in 3D Slicer (tangential linear distances to axes).

Table 1. Mean linear distance differences (in $\mathrm{mm}$ ) between two point markers of SimPlant Pro (Manual registration (MR)) and MeshLab and $3 D$ Slicer (semiautomatic registration (SR)) in comparison to physical measurement.

\begin{tabular}{cc}
$\begin{array}{c}\text { Manual } \\
\text { Registration } \\
\text { (MR) }\end{array}$ & $\begin{array}{c}\text { Semi-automatic } \\
\text { Registration (SR) }\end{array}$ \\
$\begin{array}{c}\text { Mean (Standard } \\
\text { deviation) }\end{array}$ & $\begin{array}{c}\text { Mean (Standard } \\
\text { deviation) }\end{array}$ \\
\hline$-0.19(0.17)$ & $-0.10(0.15)$ \\
\hline
\end{tabular}

\begin{tabular}{rll}
\hline A & $-0.21(0.12)$ & $-0.16(0.12)$ \\
\hline B & $-0.13(0.11)$ & $-0.05(0.10)$ \\
\hline C & $-0.29(0.13)$ & $-0.18(0.15)$ \\
\hline D & $-0.21(0.16)$ & $-0.10(0.14)$ \\
\hline E & $-0.17(0.22)$ & $-0.06(0.17)$ \\
\hline F & $-0.14(0.19)$ & $-0.07(0.18)$ \\
\hline Plane & &
\end{tabular}

Z coord

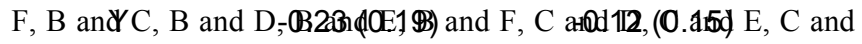
$\mathrm{F} ;\left(P<\mathrm{Z}^{0.008}\right) . \quad-0.19(0.19) \quad-0.17(0.18)$

The root-mean-square (RMS) of the tangential distances to the (x, y, z)-axes i.e. target registration error (TRE) of MR and SR were found to be $0.56 \mathrm{~mm}$ (SD 0.24) and $0.39 \mathrm{~mm}$ (SD 0.21) respectively (Table 2). No significant differences in TRE was found for MR $(P<0.05)$. For SR, first measurement block B and $\mathrm{C}, \mathrm{C}$ and $\mathrm{D}, \mathrm{C}$ and $\mathrm{E}, \mathrm{C}$ and $\mathrm{F} ;(P<0.008)$.

\section{Discussion}

The use of a CC as a surgical stereotactic marker is novel in the surgical field. Our preliminary study [13] was based on one cube and only manual registration (MR) in SimPlant Pro was performed. This provided standard deviation for estimation of sample size required for equivalence test in this study. found in this study were relatively small in comparison to previous studies. Pinsky et al. [15] and Lagravere et al. [7] found $0.27 \mathrm{~mm}$ and $1.0 \mathrm{~mm}$ mean linear measurement error in CBCT respectively. Fitzpatrick et al. [9] revealed $0.59,0.44$ and $0.30 \mathrm{~mm}$ TRE respectively when 10,20 and 50 fiducial points were used for point-based registration. Therefore, the use of $\mathrm{CC}$ as a surgical stereotactic marker in CBCT is supported by this study because it has an advantage of an easy calibration by physical measurement.

With adequate sample size, plots of the differences are presented in the present study (Figures 4 - 6). For MR, the intercept $=-0.072$ (significant, $P=0.019$ ), slope $=-0.004$ $(\mathrm{P}<0.001), R-$ squared $=0.087$ and for $\mathrm{SR}$, the intercept $=$ -0.082 (significant, $\mathrm{P}=0.005)$, slope $=-0.001(P=0.484$, insignificant), $\quad R$-squared $=0.003$. Therefore, both registration methods have systematic longer distance than physical measurement (significant negative difference intercept; though this error is not large, around -0.072 to -0.082 ). Most datapoints are within the upper and lower bounds in the Bland and Altman plots. Meanwhile, SR is better than MR since MR would have even larger distance error when the physical length increased (significant negative slope).

\section{Conclusion}

Six plastic blocks were undergone a CBCT scan and a MR and SR registration via CC's fiducial markers. Physical distances among target markers and physical distances between target markers and $\mathrm{x} / \mathrm{y} / \mathrm{z}$-axis. Then, they were regarded as golden standard. Afterward, virtual distances and coordinates of target markers in SimplantPro and Meshlab were measured for MR and $\mathrm{SR}$, respectively. After Bland and Atman plots, the registration accuracy was validated. In evaluation, SR was found to be more accurate than MR. Both SR and MR will not induce more errors when the target point is far away from the CC's fiducial marker. Our future work is to apply both MR and SR because they are clinically applicable, for which both of them have less than $2 \mathrm{~mm}$ error.

\section{Acknowledgement}

We would like to express our gratitude to Miss Samantha Li Kar Yan, Senior Technical Officer of the Faculty of Dentistry, HKU for her statistical advices. 
Table 2. Mean coordinate differences (in $\mathrm{mm}$ ) of point markers measured in SimPlant Pro (Manual registration (MR)) and MeshLab and 3D Slicer (semi-automatic registration (SR)) in comparison to physical measurement.

\begin{tabular}{|c|c|c|c|c|c|c|c|c|}
\hline & \multicolumn{4}{|c|}{ Manual Registration (MR) } & \multicolumn{4}{|c|}{ Semi-automatic Registration (SR) } \\
\hline & \multicolumn{4}{|c|}{ Mean (Standard deviation) } & \multicolumn{4}{|c|}{ Mean (Standard deviation) } \\
\hline & $\begin{array}{c}\text { Discrepancy } \\
\text { in X } \\
\text { coordinate }\end{array}$ & $\begin{array}{c}\text { Discrepancy } \\
\text { in Y } \\
\text { coordinate }\end{array}$ & $\begin{array}{c}\text { Discrepancy } \\
\text { in } Z \\
\text { coordinate }\end{array}$ & $\begin{array}{c}\text { Root mean } \\
\text { square error } \\
\text { (RMS) }\end{array}$ & $\begin{array}{c}\text { Discrepancy } \\
\text { in X } \\
\text { coordinate }\end{array}$ & $\begin{array}{c}\text { Discrepancy } \\
\text { in Y } \\
\text { coordinate }\end{array}$ & $\begin{array}{c}\text { Discrepancy } \\
\text { in } Z \\
\text { coordinate }\end{array}$ & $\begin{array}{c}\text { Root mean } \\
\text { square error } \\
\text { (RMS) }\end{array}$ \\
\hline Overall & $0.21(0.18)$ & $0.35(0.17)$ & $0.41(0.21)$ & $0.56(0.24)$ & $-0.02(0.22)$ & $0.10(0.19)$ & $-0.08(0.36)$ & $0.39(0.20)$ \\
\hline \multicolumn{9}{|l|}{ Block } \\
\hline $\mathrm{A}$ & $0.34(0.05)$ & $0.29(0.19)$ & $0.52(0.27)$ & $0.55(0.33)$ & $-0.19(0.19)$ & $-0.17(0.34)$ & $-0.23(0.19)$ & $0.37(0.20)$ \\
\hline $\mathrm{B}$ & $0.29(0.20)$ & $0.43(0.14)$ & $0.45(0.14)$ & $0.71(0.22)$ & $0.17(0.22)$ & $0.27(0.11)$ & $-0.17(0.19)$ & $0.36(0.13)$ \\
\hline $\mathrm{C}$ & $0.24(0.22)$ & $0.45(0.14)$ & $0.39(0.20)$ & $0.64(0.22)$ & $0.18(0.20)$ & $0.39(0.23)$ & $-0.64(0.20)$ & $0.65(0.23)$ \\
\hline $\mathrm{D}$ & $0.10(0.23)$ & $0.40(0.27)$ & $0.26(0.20)$ & $0.54(0.23)$ & $0.22(0.19)$ & $0.20(0.27)$ & $0.17(0.20)$ & $0.38(0.15)$ \\
\hline $\mathrm{E}$ & $0.14(0.13)$ & $0.26(0.11)$ & $0.34(0.10)$ & $0.48(0.15)$ & $-0.12(0.17)$ & $0.11(0.13)$ & $0.17(0.13)$ & $0.23(0.15)$ \\
\hline $\mathrm{F}$ & $0.17(0.11)$ & $0.30(0.12)$ & $0.47(0.23)$ & $0.48(0.20)$ & $-0.24(0.13)$ & $0.03(0.19)$ & $0.31(0.12)$ & $0.36(0.11)$ \\
\hline
\end{tabular}

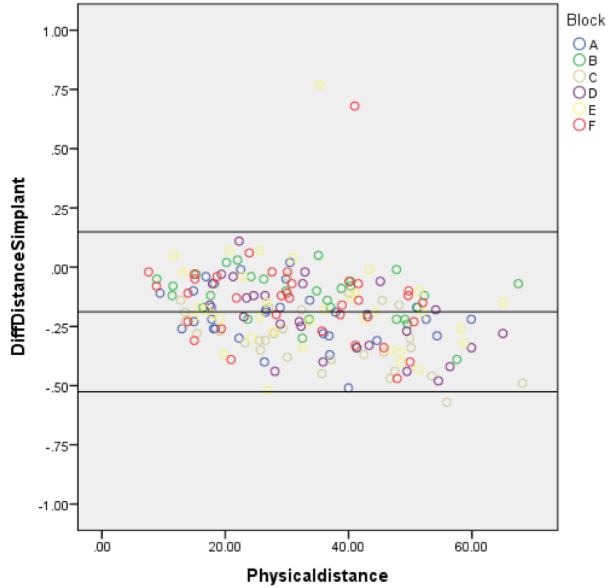

(a) horizontal lines representing mean - 1.96 SD (lower) mean (middle), and mean + 1.96 SD (upper). Remark: DiffDistanceSimplant (MR), DiffDistanceMeshlab $(S R)$, Physicaldistance $=$ distance between two markers

\section{References}

[1] Buser D, Martin W, Belser UC. Optimizing esthetics for implant restorations in the anterior maxilla: anatomic and surgical considerations. Int J Oral Maxillofac Implants 2004;19 Suppl:43-61.

[2] Hatcher DC. Operational principles for cone-beam computed tomography. J Am Dent Assoc 2010;141 Suppl 3:3S-6S.

[3] Wang G, Crawford CR, Kalender WA. Multirow detector and conebeam spiral/helical CT. IEEE Trans. Med. Imag. 2000;19(9):817-21.

[4] Verhamme LM, Meijer GJ, Boumans T, Schutyser F, Berge SJ, Maal TJ. A clinically relevant validation method for implant placement after virtual planning. Clin Oral Implants Res 2013;24(11):1265-72.

[5] Maes F, Collignon A, Vandermeulen D, Marchal G, Suetens P. Multimodality image registration by maximization of mutual information. IEEE Trans. Medical Imaging 1997;16(2):187-98.

[6] Hill DLG, Batchelor PG, Holden M, Hawkes DJ. Medical image registration. Physics in Medicine and Biology 2001;46(3):R1-R45.

[7] Lagravere MO, Carey J, Toogood RW, Major PW. Three-dimensional accuracy of measurements made with software on cone-beam

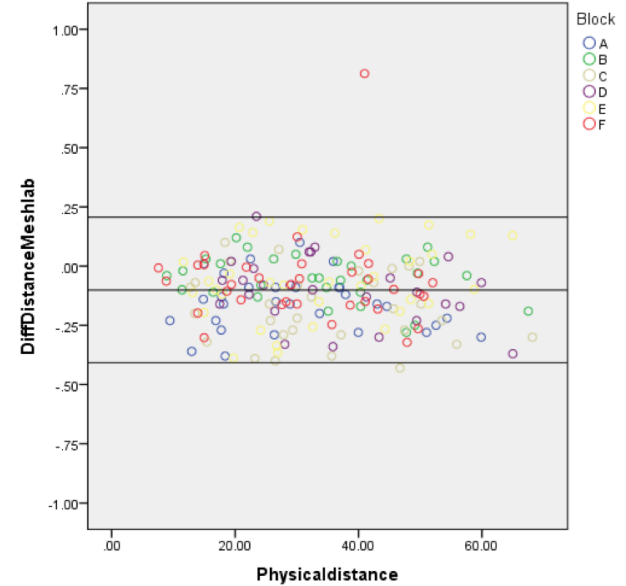

b)

computed tomography images. Am $J$ Orthod Dentofacial Orthop 2008;134(1):112-6.

[8] Hassfeld S, Muhling J. Computer assisted oral and maxillofacial surgery--a review and an assessment of technology. Int $J$ Oral Maxillofac Surg 2001;30(1):2-13.

[9] Fitzpatrick JM, West JB, Maurer CR. Predicting error in rigid-body point-based registration. IEEE Trans. Med. Imag. 1998;17(5):694-702.

[10] Maurer CR, Fitzpatrick JM, Wang MY, Galloway RL, Maciunas RJ, Allen GS. Registration of head volume images using implantable fiducial markers. IEEE Trans. Medical Imaging 1997;16(4):447-62.

[11] Grimson WEL, Ettinger GJ, White SJ, LozanoPerez T, Wells WM, Kikinis R. An automatic registration method for frameless stereotaxy, image guided surgery, and enhanced reality visualization. IEEE Trans. Medical Imaging 1996;15(2):129-40.

[12] Greenstein G, Tarnow D. The mental foramen and nerve: clinical and anatomical factors related to dental implant placement: a literature review. J Periodontol 2006;77(12):1933-43.

[13] Lam WY, Ngan HY, Wat PY, Luk HW, Pow EH, Goto TK. Novel geometric coordination registration in cone-beam computed Tomogram. IEEE AIPR Workshop, 2014: p. 1-6.

[14] Bartlett JW, Frost C. Reliability, repeatability and reproducibility: analysis of measurement errors in continuous variables. Ultrasound in Obstetrics \& Gynecology 2008;31(4):466-75. 
[15] Pinsky HM, Dyda S, Pinsky RW, Misch KA, Sarment DP. Accuracy of three-dimensional measurements using cone-beam CT. Dentomaxillofac Radiol 2006;35(6):410-6.

[16] [online] Meshlab, http://meshlab.sourceforge.net/

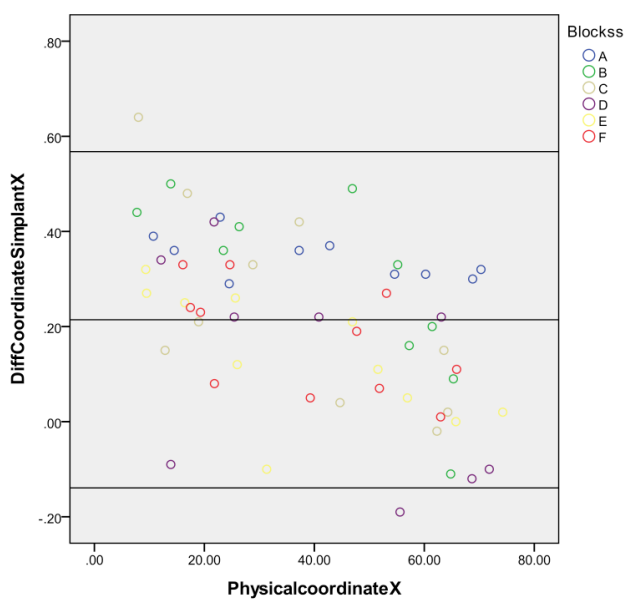

(a)

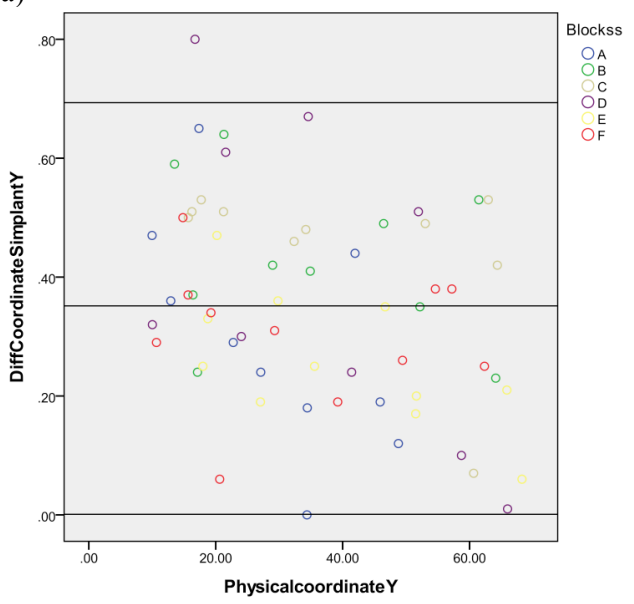

(b)

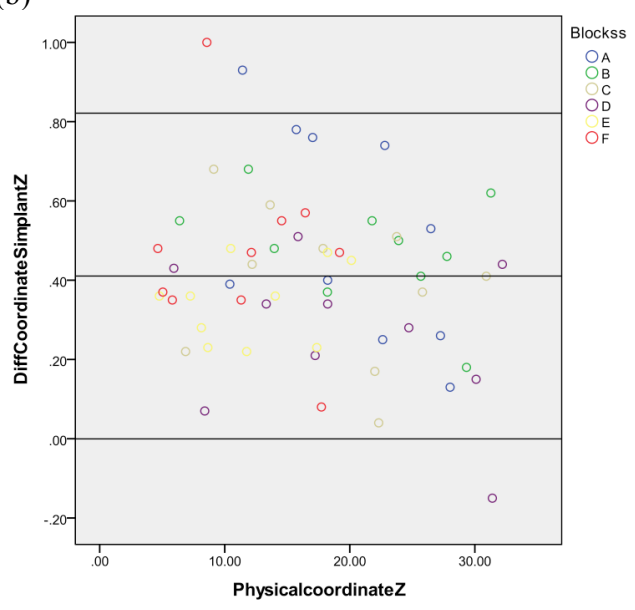

(c)

Figure 5. Bland and Altman plots of the differences in coordinate SimPlant Pro to physical measurement, (a) axis $x$, (b) axis $y$, (c) axis $z$, with the horizontal lines representing mean - 1.96 SD (lower) mean (middle), and mean + $1.96 \mathrm{SD}$ (upper). Remark: DiffDistanceSimplant (MR), DiffDistanceMeshlab (SR) Physicalcoordinate $X / Y / Z$ = distance between one marker to $X / Y / Z$ -
[17] [online] Slicer, http://slicer.org/

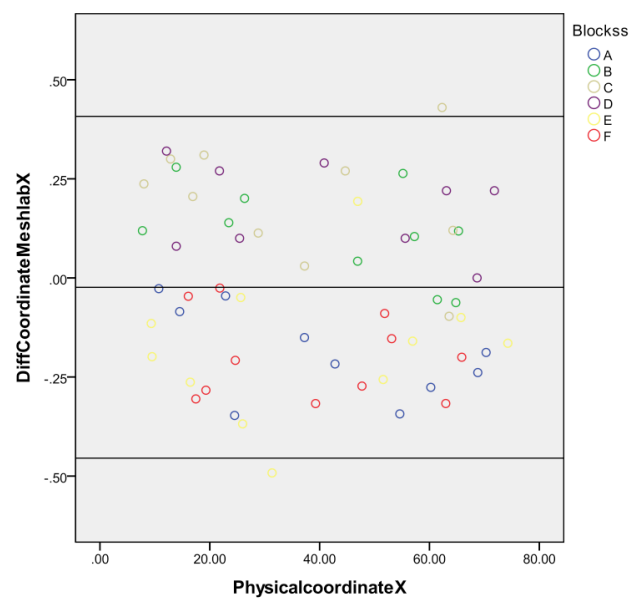

(a)

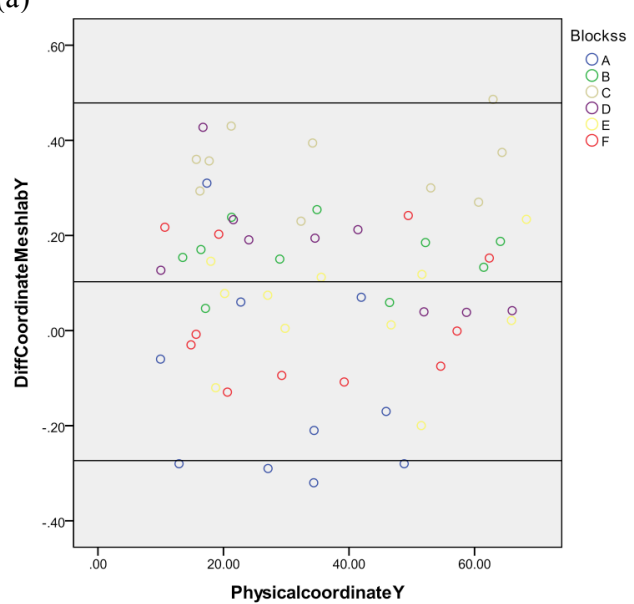

(b)

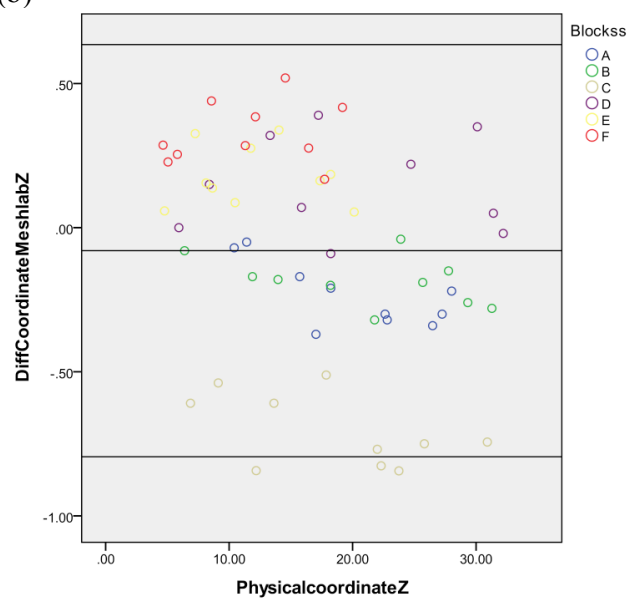

(c)

Figure 6. Bland and Altman plots of the differences in coordinate MeshLab and 3D Slicer to physical measurement, (a) axis $x$, (b) axis $y$, (c) axis $z$, with the horizontal lines representing mean - $1.96 S D$ (lower) mean (middle), and mean + 1.96 SD (upper). Remark: DiffDistanceSimplant (MR), DiffDistanceMeshlab (SR) Physicalcoordinate $X / Y / Z$ = distance between one marker to $X / Y / Z$ axis. 